JGG 2020;68:231-234

doi: $10.36150 / 2499-6564-258$

\title{
Could ischemic colitis be the first manifestation of COVID-19? A case report
}

\author{
Paola Giustina Simeone ${ }^{1-3}$, Francesca Crosta ${ }^{2-4}$, Donatella Stanziani ${ }^{2,3}$, \\ Franco Colameco ${ }^{2,3}$, Carlo Sanrocco ${ }^{3}$, Giovambattista Desideri ${ }^{5}$, \\ Francesca Santilli ${ }^{2}$, Vincenzo Savini ${ }^{4}$, Rosa Scurti2,3, Giustino Parruti ${ }^{3}$ \\ ${ }^{1}$ Department of Medicine and Aging, Center for Advanced Studies and Technology (CAST), \\ University "G. d'Annunzio" of Chieti-Pescara, Chieti, Italy; ${ }^{2}$ Department of Geriatric Unit, Azienda \\ Sanitaria Locale (AUSL) di Pescara, Pescara, Italy; ${ }^{3}$ Department of Infectious Disease, Azienda \\ Sanitaria Locale (AUSL) di Pescara, Italy; ${ }^{4}$ Clinical Microbiology and Virology, Spirito Santo Hospital, \\ Pescara, Italy; ${ }^{5}$ Department of Life, Health \& Environmental Sciences, University of L'Aquila, Italy
}

We report on a case of SARS-CoV-2-infected patient with clinical and histologic features mimicking ischaemic colitis. This case provides evidence that SARS-CoV-2 may compromise the microvascular blood flow in the intestinal wall, with a parallel activation of the inflammatory cascade, either in the absence, or earlier of any pulmonary involvement.

Key words: SARS-CoV-2, inflammation, ischaemic colitis, microthrombi

\section{CASE-PRESENTATION}

Received: August 28, 2020

Accepted: November 27, 2020

\section{Correspondence \\ Francesca Crosta}

Department of Life, Health \& Environmental Sciences, University of L'Aquila, piazzale S. Tommasi, 67100 Coppito-L'Aquila, Italy. E-mail: francydoc@gmail.com

\section{Conflict of interest}

The Authors declare no conflict of interest

How to cite this article: Simeone PG, Crosta F, Stanziani $D$, et al. Could ischemic colitis be the first manifestation of COVID-19? A case report. Journal of Gerontology and Geriatrics 2020;68:231-4. https://doi.org/10.36150/2499-6564-258

(C) Copyright by Società Italiana di Gerontologia e Geriatria (SIGG)

\section{(c) (1) (9) $(9)$}

\section{OPEN ACCESS}

This is an open access article distributed in accordance with the CC-BY-NC-ND (Creative Commons Attribution-NonCommercial-NoDerivatives 4.0 International) license. The article can be used by giving appropriate credit and mentioning the license, but only for non-commercial purposes and only in the original version. For further information: https://creativecommons.org/licenses/by-nc-nd/4.0/deed.en
A 87-year-old man with a 14-day history of abdominal pain, diarrhoea and rectal bleeding was admitted to the Geriatric Unit of Pescara General Hospital on April 27 ${ }^{\text {th }}$ 2020, in a no COVID department, and the patient was hospitalized in a single room. The patient had a history of non-metastatic prostate cancer, treated with prostatectomy ten years in advance. He was a former smoker. He was cachectic and sarcopenic. He took no drugs at home.

Ten days before admission the patient had a stool culture test, performed a parasite stool test, both negative, paralleled by a negative search for Clostridium Difficile antigen and toxin. Chemical-physical examination of stools revealed the presence of leucocytes (25-30 pcv)

At the emergency department blood pressure was $90 / 40 \mathrm{mmHg}$, oxygen saturation $97 \%$ on air, breath rate $10 /$ minute and temperature $36^{\circ} \mathrm{C}$.

Biochemical sampling revealed leucocytosis with lymphocytopenia (14.7 $\times 10^{3} / \mathrm{LL}$, percentage of neutrophils $91.8 \%$ and lymphocytes $(5.1 \%)$ ), haemoglobin $10.5 \mathrm{~g} / \mathrm{dL}$, high levels of D-DIMER (23 mg/L), INR 1.3, lactate dehydrogenase $(\mathrm{LDH}) 568 \mathrm{mg} / \mathrm{L}$, pro-calcitonin $0.99 \mathrm{ng} / \mathrm{mL}$, glucose 234 $\mathrm{mg} / \mathrm{dL}$, blood urea nitrogen (BUN) $82 \mathrm{mg} / \mathrm{dL}$, creatinine $1.84 \mathrm{mg} / \mathrm{dL}$, and sodium $122 \mathrm{mEq} / \mathrm{L}$ (Fig. 1, panel A-B). A CT scan of the abdomen showed moderate thickening of colonic walls. Infectious and surgical consultancies were requested. Oropharyngeal and nasopharyngeal swabs for SARSCoV-2 were negative.

On admission, rectoscopy was immediately executed and revealed an endoscopic pattern suggestive of ischemic colitis (Fig. 2, panel B). Biopsy specimens were collected and the histological characterization showed the presence of dense lymphoplasmacellular and granulocytic infiltrates, lining to the lamina propria and muscularis mucosae of the recto-sigmoidal 


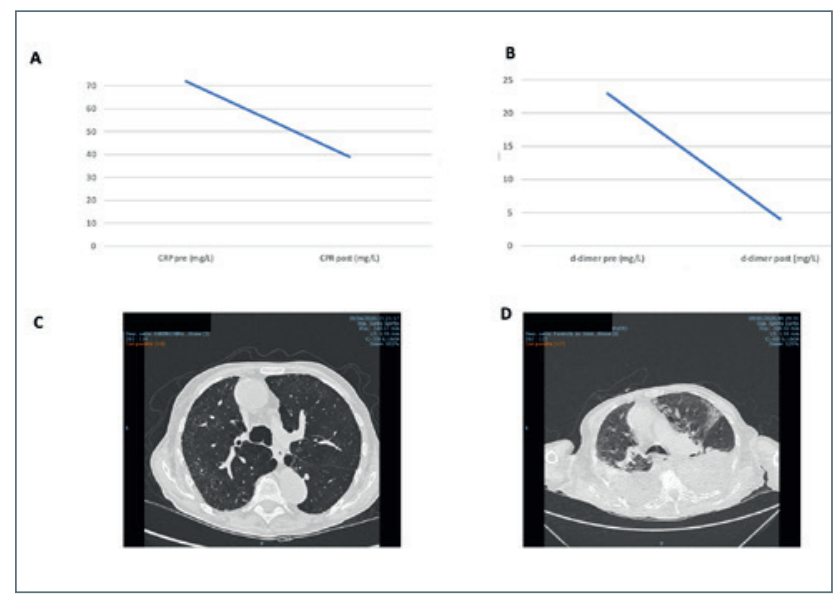

Figure 1. A time-course showing sampling and exams before and during hospitalization; (A chest CT scan, with multiple patchy/ground glass shadows in both lungs).

junction. Infiltrates appeared to be composed of lymphoid aggregates and immunohistochemically polytypic plasmacells. There were rare cryptic micro-abscesses, with micro-erosive aspects, hyaline microthrombi in the capillaries and hyperplastic-regenerative modifications of the crypt epithelium.

Histological findings were suggestive of active colitis with a likely infective pathogenesis (Fig. 2, panel A).

The search for alert germs on stool specimens was negative. Serologic tests for Cytomegalovirus and Epstein barr virus antibodies (IgM and IgG) were negative. Four days later, the patient developed chills and fever, with a maximum body temperature of $38.5^{\circ} \mathrm{C}$ associated with dyspnoea and shortness of breath. Urine volume was reduced, and oxygen saturation was 85\%, while on ambient air.

Oxygen was delivered through a nonrebreather face mask, at a rate of 6 liters per minute; oxygen saturation rose to $94 \%$.

Legionella pneumophila antigen, Mycoplasma pneumoniae and Chlamydia pneumonia antibodies were negative. Interleukin-6 levels were elevated (148.67 pg/mL).

A chest CT scan, performed after ten days, revealed multiple patchy/ground glass shadows in both lungs (fig. 1, panel D).

A second real time reverse - transcriptase-polymerase chain reaction (rRT-PCR) for SARS-CoV-2 from a pharyngeal sample was again negative, as well as IgG and IgM antibody repeated assays.

We decided to search for SARS-CoV-2 virus in stool samples, taken on admission. This search provided positive results, at variance with previous collections. A $2 \mathrm{~mm}$-diameter stool aliquot was diluted into $1 \mathrm{~mL}$ physiologic steril solution; the mixture was vortexed

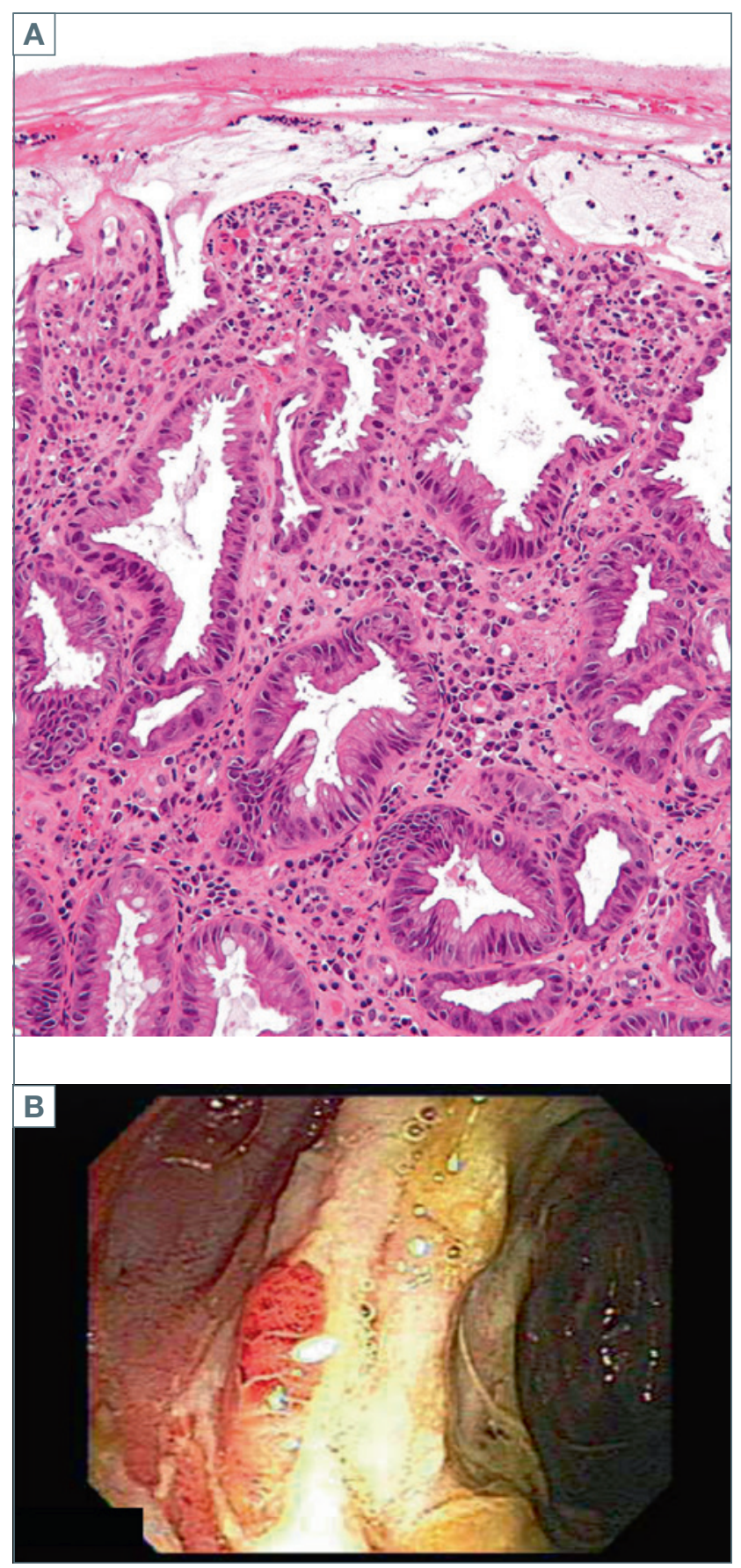

Figure 2. A representative endoscopic image of the colon and histology, showing vascular alterations

(10 seconds), then centrifugated at $5000 \mathrm{rpm}$ (7 seconds); a $50 \mu \mathrm{L}$ surnatant aliquot was introduced into the collection buffer provided in the Credo Diagnostics VitaPCR $^{\circledR}$ SARS-CoV-2 Assay package (A. Menarini Diagnostics s.r.l., Firenze, Italy). The assay was then performed according to the manufacturer's instructions for naso/oropharyngeal swabs by using the VitaPCR ${ }^{\mathrm{TM}}$ 
Instrument (A. Menarini Diagnostics s.r.l.); the latter is a rapid molecular platform based on RT-PCR amplification technology targeting two different regions of the SARS-CoV-2 N gene (a SARS-CoV-2-specific region, as well as a region shared by all Sarbecoviruses). Both molecular targets were amplified providing conclusive evidence of SARS-CoV-2 infection.

The oropharyngeal and nasopharyngeal swabs for SARS-CoV-2, once more repeated after another day, turned out positive.

The patient was treated with hydroxychloroquine, clarithromycin, high dose corticosteroids and heparin.

Respiratory failure ensued, requiring first high-flow oxygen and subsequently mechanical ventilation.

Unfortunately, the patient's condition rapidly worsened for superimposed pulmonary bacterial Pseudomonas Aeruginosa infection rapidly evolving into septic shock, complicated with disseminated intravascular coagulopathy (DIC). The patient died on May 13.

\section{DISCUSSION}

The majority of patients with SARS-CoV-2 infection have respiratory symptoms ${ }^{1}$. However, in a cohort of 140 COVID-19 patients in Wuhan, gastrointestinal symptoms were described in up to $39.6 \%$ of patients, including nausea in 24 (17.3\%), diarrhoea in 18 (12.9\%), and vomiting in $7(5.0 \%)^{2}$. Similarly, the rate of diarrhoea was up to $35.6 \%$ in another cohort of 73 patients ${ }^{2}$. On the other hand, abdominal pain was rarely described, being reported in $2.2-5.8 \%$ in patients ${ }^{3}$.

Evidence from previous studies indicated that coronaviruses have a tropism to the gastro-intestinal (GI) tract ${ }^{4,5}$. Thus Gl tropism may explain the frequent occurrence of diarrhoea in coronavirus infection ${ }^{5}$.

Hence, diarrhoea can be the initial symptom and may occur earlier than pyrexia or respiratory distress in some cases. However, the case we report on is unique in that Gl symptoms occurred in the absence of other typical COVID-19 features. These symptoms were clinically relevant inducing an early endoscopic evaluation led to an apparently sound diagnosis of ischemic colitis thus hindering early diagnosis of SARS-CoV-2.

This peculiar GI manifestation may be, at least in part, a reflection of the microvascular thrombosis of the colic mucosa vessels induced by SARS-CoV-2 infection. Indeed, is well known that patients with COVID-19 are at high risk of developing thromboembolic complications ${ }^{6}$. Widespread thrombosis with microangiopathy is increasingly revealed by autopsy studies ${ }^{7}$. Patients with COVID-19 fulfil at least two out of three criteria of Virchow's triad - reduced venous flow from immobility and prothrombotic changes due to inflammatory state. There is increasing evidence to suggest that the third criterium of Virchow's triad - vessel wall changes - may also exist in patients with COVID-19 ${ }^{8}$. Endothelial damage and subsequent clotting in the small vessels is common in severe and critical COVID-19, and may involve all organs besides the lungs, including the heart, liver, kidney and probably, as in our case, intestine. This may have implications for treatment ${ }^{8,9}$. The efficacy and safety of anticoagulants in this setting is unknown.

In Xiao et al. study ${ }^{3}$, the age of patients with positive results for SARS-CoV-2 RNA in stool ranged from 10 months to 78 years old. The cause of the different presentation is an interesting matter. Factors such as genetics, age, and immunological response could be relevant explanations ${ }^{10}$.

Clinicians must be aware of the possibility of COVID-19 presenting nonspecifically, so cases are not missed when they fall outside current diagnostic and management guidelines ${ }^{11}$. This has implications for the diagnosis and isolation of COVID-19 in frail older people ${ }^{11}$. Gan's study ${ }^{12}$ demonstrated that 40\% (49/122) of older population (mean age $81 \pm 8 \mathrm{SD}$ ) with COVID-19 had atypical presenting complaints in the community. Although patients with atypical presentation appeared to have worse outcomes. The rate of atypical presentations of COVID-19 observed was similar to that found by Hofman and colleagues in 2017 for a general medical patient cohort aged $>80$ years presenting to an emergency department ${ }^{12}$.

The early intestinal manifestation, coupled with the two negative oropharyngeal and nasopharyngeal swabs for SARS-CoV-2, delayed the diagnosis. This led to a rapid spread of COVID-19 in the ward and worsened the prognosis.

\section{CONCLUSIONS}

This case report provides preliminary evidence of intestinal mucosa involvement by inflammatory and ischemic processes, leading to clinical manifestations that mimic ischemic colitis early in a lethal COVID-19 infection.

Diarrhoea and rectal bleeding significantly anteceded respiratory symptoms with a positive faecal sample ten days in advance of positive oropharyngeal and nasopharyngeal swabs.

The diagnosis was made possible by the search of SARS-CoV-2 in a stool specimen collected on admission. Our experience may be useful to set up thorough microbiological characterization of uncertain cases.

\section{ACKNOWLEDGMENTS:}

We are grateful to Clinical and Microbiological staff of Pescara General Hospital, particularly for assistance in diagnosis. 


\section{References}

1 Chen N, Zhou M, Dong X, et al. Epidemiological and clinical characteristics of 99 cases of 2019 novel coronavirus pneumonia in Wuhan, China: adescriptive study. Lancet 2020;395:507-13.

2 Zhang JJ, Dong X, Cao YY, et al. Clinical characteristics of 140 patients infected with SARS-CoV-2 in Wuhan, China. Allergy 2020;Feb 19. https://doi.org/10.1111/ all.14238. [Epub ahead of print]

3 Xiao F, Tang M, Zheng X, et al. Evidence for gastrointestinal infection of SARS-CoV-2. Gastroenterology 2020;158:1831-3.e3.

4 Liang W, Feng Z, Rao S, et al. Diarrhea may be underestimated: a missing linking 2019 novel coronavirus. Gut 2020;69:1141-3.

5 Wong SH, Lui RN, Sung JJ. COVID-19 and the digestive system. J Gastroenterol Hepatol 2020 35:744-8.

6 Walls AC, Park YJ, Tortorici MA, et al. Structure, function, and antigenicity of the SARS-CoV-2 spike glycoprotein. Cell 2020;181:281-92.e6.

7 Ackermann M, Verleden SE, Kuehnel M, et al. Pulmonary vascular endothelialitis, thrombosis, and angiogenesis in COVID-19. N Engl J Med 2020;May 21. https://doi. org/10.1056/NEJMoa2015432

8 Connors JM, Levy JH. COVID-19 and its implications for thrombosis and anticoagulation. Blood 2020;Apr 27:blood.2020006000.

9 Guo J, Huang Z, Lin L, et al. Coronavirus disease 2019 (COVID-19) and cardiovascular disease: a viewpoint on the potential influence of angiotensin-converting enzyme inhibitors/angiotensin receptor blockers on onset and severity of severe acute respiratory syndrome coronavirus 2 infection. J Am Heart Assoc 2020;9:e016219.

10 Wahab SF, Løgstrup BB. Atypical manifestations of COVID-19 in general practice: a case of gastrointestinal symptoms. BMJ Case Rep 2020;13:e237520. https://doi. org/10.1136/bcr-2020-237520

11 Tay HS, Harwood R. Atypical presentation of COVID-19 in a frail older person. Age Ageing 2020;49:523-4. https:// doi.org/10.1093/ageing/afaa068

12 Gan JM, Kho J, Akhunbay-Fudge M, et al. Atypical presentation of COVID-19 in hospitalised older adults. Ir J Med Sci 2020;1-6. https://doi.org/10.1007/s11845-02002372-7 